Appl. Set-Valued Anal. Optim. 1 (2019), No. 2, pp. 95-103

Available online at http://asvao.biemdas.com

https://doi.org/10.23952/asvao.1.2019.2.01

\title{
A RANDOM TOPOLOGICAL FIXED POINT INDEX FOR A CLASS OF MULTIVALUED MAPS
}

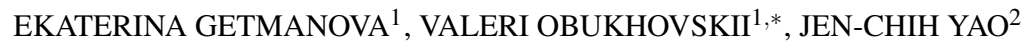 \\ ${ }^{1}$ Department of Higher Mathematics, Voronezh State Pedagogical University, Voronezh, Russia \\ ${ }^{2}$ Center for General Education, China Medical University, Taichung 40402, Taiwan
}

\begin{abstract}
We present the construction of the topological fixed point index for a class of random condending compositions of multimaps with aspheric values. We describe its main properties and give an application to random fixed points.

Keywords. Condensing map; Random multimap; Random fixed point; Random fixed point index.
\end{abstract}

2010 Mathematics Subject Classification. 47H11, 47H04, 47H08.

\section{INTRODUCTION}

Currently the development of the fixed point theory for random single-valued and multivalued maps plays an important role in the study of nondeterministic systems (see, for example, [1], [3], [6], [7], [8], [10], [13] - [16] and the references therein). The methods of this theory find important applications in the investigation of random differential equations and inclusions. Some versions of the random topological degree theory can be found in [1], [7] and [15].

In this paper, we suggest the construction of the topological fixed point index for a class of random condending compositions of multimaps with aspheric values and give an application to random fixed points of such multimaps.

The paper is organized in the following way. Necessary preliminaries from the theory of multivalued, random and condensing maps and from the fixed point index theory are collected in the next section. In the third section, we present the main construction of the random fixed point index, describe its properties and give some application to random fixed points.

\section{PRELIMINARIES}

2.1. Multivalued maps. We describe some notions of the theory of multivalued maps that will be used in the sequel (details can be found, e.g. in [4], [7], [8] and [11]).

Let $X$ and $Y$ be metric spaces. By the symbol $C(Y)[K(Y)]$, we denote the collection of all nonempty closed [respectively, compact] subsets of $Y$.

\footnotetext{
${ }^{*}$ Corresponding author.

E-mail addresses: ekaterina_getmanova@bk.ru (E. Getmanova), valerio-ob2000@mail.ru (V.Obukhovskii), yaojc@mail. cmu.edu.tw (J.C. Yao).

Received June 13, 2019; Accepted July 8, 2019.
}

(C)2019 Applied Set-Valued Analysis and Optimization 
Definition 2.1. A multivalued map (multimap) $\mathscr{F}: X \rightarrow C(Y)$ is said to be upper semicontinuous (u.s.c.) if, for every open set $V \subset Y$,

$$
\mathscr{F}^{-1}(V)=\{x \in X: \mathscr{F}(x) \subset V\}
$$

is an open subset of $X$.

Let us mention the following properties of u.s.c. multimaps.

Lemma 2.1. Let $\mathscr{F}: X \rightarrow K(Y)$ be a u.s.c. multimap. If $A \subset X$ is a compact set, then its image $\mathscr{F}(A)$ is a compact subset of $Y$.

Lemma 2.2. Let $X, Y$ and $Z$ be metric spaces. Let $\mathscr{F}_{0}: X \rightarrow K(Y)$ and $\mathscr{F}_{1}: Y \rightarrow K(Z)$ be u.s.c. multimaps. Then the composition $\mathscr{F}_{1} \circ \mathscr{F}_{0}: X \rightarrow K(Z)$ is a u.s.c. multimap.

Definition 2.2. A multimap $\mathscr{F}: X \rightarrow K(Y)$ is said to be compact if its range $\mathscr{F}(X)$ is a relatively compact subset of $Y$.

Let $(\Omega, \Sigma)$ be a complete measurable space.

Definition 2.3. A multimap $\mathscr{F}: \Omega \rightarrow C(Y)$ is said to be measurable if $\mathscr{F}^{-1}(V) \in \Sigma$ for every open set $V \subset Y$.

Let $X, Y$ be separable metric spaces.

Definition 2.4. A multimap $\mathscr{F}: \Omega \times X \rightarrow C(Y)$ is called a random $u$-multimap provided that

(i) $\mathscr{F}$ is measurable with respect to a minimal $\sigma$-algebra generated by $\Sigma \times B(X)$, where $B(X)$ is the collection of all Borel subsets of $X$;

(ii) for every $\omega \in \Omega$, the multimap $\mathscr{F}(\omega, \cdot): X \rightarrow C(Y)$ is u.s.c.

Definition 2.5. Let $A \subseteq X$ be a closed set. A measurable map $\xi: \Omega \rightarrow A$ is called a random fixed point of a multimap $\mathscr{F}: \Omega \times A \rightarrow C(X)$ if

$$
\xi(\omega) \in \mathscr{F}(\omega, \xi(\omega))
$$

for all $\omega \in \Omega$.

Proposition 2.1. ([7], Proposition 31.3). Let $\mathscr{F}: \Omega \times A \rightarrow C(X)$ be a random u-multimap such that, for every $\omega \in \Omega$, the set of fixed points

$$
\text { Fix } \mathscr{F}(\omega, \cdot)=\{x \in X: x \in \mathscr{F}(\omega, x)\}
$$

is nonempty. Then $\mathscr{F}$ has a random fixed point.

2.2. $J^{c}$-multimaps. To present the class of multimaps which will be considered, we recall some notions.

Given a subset $A$ of a metric space $Y$ and $\varepsilon>0$, we denote by $O_{\varepsilon}(A)$ the $\varepsilon$-neighborhood of $A$.

Definition 2.6. (see, e.g., [4], [7] and [12]) A nonempty compact subset $A$ of a metric space $Y$ is said to be aspheric (or $U V^{\infty}$, or $\infty$-proximally connected) if for every $\varepsilon>0$, there exists $\delta, 0<\delta<\varepsilon$ such that, for each $n=0,1,2, \ldots$, every continuous map $g: S^{n} \rightarrow O_{\delta}(A)$ can be extended to a continuous map $\tilde{g}: B^{n+1} \rightarrow O_{\varepsilon}(A)$, where $S^{n}=\left\{x \in \mathbb{R}^{n+1}:\|x\|=1\right\}$ and $B^{n+1}=\left\{x \in \mathbb{R}^{n+1}:\|x\| \leq 1\right\}$.

Definition 2.7. (see [9]) A nonempty compact space $A$ is said to be an $R_{\delta}$-set if it can be represented as the intersection of a decreasing sequence of compact, contractible spaces. 
Definition 2.8. A u.s.c. multimap $\mathscr{F}: X \rightarrow K(Y)$ is said to be a $J$-multimap $(\mathscr{F} \in J(X, Y)$ ) if every value $\mathscr{F}(x), x \in X$ is an aspheric set.

Now let us recall (see, e.g., [5], [7]) that a metric space $Z$ is called the absolute retract (the $A R$-space) [resp., the absolute neighborhood retract (the $A N R$-space)] provided that for each homeomorphism $h$ taking it onto a closed subset of a metric space $Z^{\prime}$, the set $h(Z)$ is the retract of $Z^{\prime}$ [resp., of its open neighborhood in $Z^{\prime}$ ]. Notice that the class of $A N R$-spaces is broad enough: in particular, a finite-dimensional compact set is the $A N R$-space if and only if it is locally contractible. In turn, it means that compact polyhedrons and compact finite-dimensional manifolds are the $A N R$-spaces. The union of a finite number of convex closed subsets in a normed space is also the $A N R$-space.

Proposition 2.2. (see [7]) Let $Z$ be an ANR-space. In each of the following cases a u.s.c. multimap $\mathscr{F}: X \rightarrow K(Z)$ is a $J$-multimap:

for each $x \in X$ the value $\mathscr{F}(x)$ is

a) a convex set;

b) a contractible set;

c) an $R_{\delta}$-set;

d) an AR-space.

In particular, every continuous map $\sigma: X \rightarrow Z$ is a J-multimap.

Definition 2.9. By $J^{c}(X, Y)$ we denote the collection of all multimaps $\mathscr{F}: X \rightarrow K(Y)$ that may be represented in the form of composition $\mathscr{F}=\mathscr{F}_{n} \circ \ldots \circ \mathscr{F}_{1}, n \geq 1$, where $\mathscr{F}_{i} \in J\left(X_{i-1}, X_{i}\right), i=1, \ldots, n$, $X_{0}=X, X_{n}=Y$, and $X_{i}$ for $0<i<n$ are open subsets of normed spaces. The composition $\mathscr{F}_{n} \circ \ldots \circ \mathscr{F}_{1}$ will be called the decomposition of $\mathscr{F}$. We will denote $D_{\mathscr{F}}=\left(\mathscr{F}_{n} \circ \ldots \circ \mathscr{F}_{1}\right)$.

It is worth noting that a multimap can admit different decompositions (see [7]).

Now, let $U$ be an open subset of a normed space $E$ and let $\mathscr{F}: \bar{U} \rightarrow K(E)$ be a compact multimap of the class $J^{c}(\bar{U}, E)$ having a decomposition

$$
D_{\mathscr{F}}: \bar{U}=X_{0} \stackrel{\mathscr{F}_{1}}{\longrightarrow} X_{1} \stackrel{\mathscr{F}_{2}}{\longrightarrow} \ldots \stackrel{\mathscr{F}_{n}}{\longrightarrow} X_{n}=E
$$

and such that $x \notin \mathscr{F}(x)$ for all $x \in \partial U$. In this situation, the topological characteristic, the fixed point index $\operatorname{ind}\left(D_{\mathscr{F}}, \bar{U}\right)$ of the decomposition $D_{\mathscr{F}}$ on $\bar{U}$ is well defined (see [2]).

Let us mention some properties of the fixed point index.

Proposition 2.3. (Existence) If ind $\left(D_{\mathscr{F}}, \bar{U}\right) \neq 0$, then $\emptyset \neq F i x \mathscr{F} \subset U$.

To describe the next property, we need the following definition.

Definition 2.10. Let $\mathscr{F}, \mathscr{G} \in J^{c}(\bar{U}, E)$ be two compact multimaps with decompositions

$$
\begin{gathered}
D_{\mathscr{F}}: \bar{U}=X_{0} \stackrel{\mathscr{F}_{1}}{\rightarrow} X_{1} \stackrel{\mathscr{F}_{2}}{\longrightarrow} \ldots \stackrel{\mathscr{F}_{n}}{\rightarrow} X_{n}=E, \\
D_{\mathscr{G}}: \bar{U}=X_{0} \stackrel{\mathscr{G}_{1}}{\rightarrow} X_{1} \stackrel{\mathscr{G}_{2}}{\rightarrow} \ldots \stackrel{\mathscr{G}_{n}}{\rightarrow} X_{n}=E,
\end{gathered}
$$

and such that $x \notin \mathscr{F}(x)$ and $x \notin \mathscr{G}(x)$ for all $x \in \partial U$. We will say that the decompositions $D_{\mathscr{F}}$ and $D_{\mathscr{G}}$ are homotopic,

$$
D_{\mathscr{F}} \sim D_{\mathscr{G}},
$$


provided that there exist multimaps

$$
\mathscr{H}_{i} \in J\left(X_{i-1} \times[0,1], X_{i}\right), \quad i=1, \ldots n
$$

with $\mathscr{H}_{i}(\cdot, 0)=\mathscr{F}_{i}, \mathscr{H}_{i}(\cdot, 1)=\mathscr{G}_{i}, i=1, \ldots, n$ and such that the multimap $\mathscr{H}: \bar{U} \times[0,1] \multimap E$ having the decomposition

$$
\bar{U} \times[0,1]=X_{0} \times[0,1] \stackrel{\mathscr{\mathscr { H }}_{1}}{\longrightarrow} X_{1} \times[0,1] \stackrel{\widetilde{\mathscr{H}}_{2}}{\longrightarrow} \ldots \stackrel{\widetilde{\mathscr{H}}_{n-1}}{\longrightarrow} X_{n-1} \times[0,1] \stackrel{\mathscr{H}_{n}}{\circ} X_{n}=E,
$$

where $\widetilde{\mathscr{H}_{i}}(x, \lambda)=\mathscr{H}_{i}(x, \lambda) \times\{\lambda\}$ for $x \in X_{i-1}, \lambda \in[0,1], i=1, \ldots, n-1$ is compact and has no fixed points on $\partial U \times[0,1]$, i.e., $x \notin \mathscr{H}(x, \lambda)$ for $x \in \partial U, \lambda \in[0,1]$.

Notice that $\mathscr{H}(\cdot, 0)=\mathscr{F}, \mathscr{H}(\cdot, 1)=\mathscr{G}$.

Proposition 2.4. (Homotopy invariance) If the decompositions $D_{\mathscr{F}}$ and $D_{\mathscr{G}}$ are homotopic, $D_{\mathscr{F}} \sim D_{\mathscr{G}}$, then

$$
\operatorname{ind}\left(D_{\mathscr{F}}, \bar{U}\right)=\operatorname{ind}\left(D_{\mathscr{G}}, \bar{U}\right) .
$$

Proposition 2.5. (Additivity) If Fix $\mathscr{F} \cap U \subset \bigcup_{j=1}^{k} U_{j}$, where $U_{j}, j=1, \ldots, k$ are open disjoint in $U$, then

$$
\operatorname{ind}\left(D_{\mathscr{F}}, \bar{U}\right)=\sum_{j=1}^{k} \operatorname{ind}\left(D_{\mathscr{F}}, \bar{U}_{j}\right) .
$$

It should be mentioned that, in general, the above defined index depends not only on $\mathscr{F}$ but also on the decomposition $D_{\mathscr{F}}$ involved.

2.3. Condensing multimaps. Let us recall some notions (see e.g. [11]). Now, let $E$ be a Banach space. Denote by $P(E)$ the collection of all nonempty subsets of $E$. Let $(\mathscr{A}, \geq)$ be a partially ordered set.

Definition 2.11. A map $\beta: P(E) \rightarrow \mathscr{A}$ is called a measure of noncompactness (MNC) in $E$ if

$$
\beta(\overline{c o} \mathscr{D})=\beta(\mathscr{D}) \text { for every } \mathscr{D} \in P(E) .
$$

A MNC $\beta$ is said to be :

(i) monotone, if $\mathscr{D}_{0}, \mathscr{D}_{1} \in P(E), \mathscr{D}_{0} \subseteq \mathscr{D}_{1}$ implies $\beta\left(\mathscr{D}_{0}\right) \leq \beta\left(\mathscr{D}_{1}\right)$;

(ii) nonsingular, if $\beta(\{a\} \cup \mathscr{D})=\beta(\mathscr{D})$ for every $a \in E, \mathscr{D} \in P(E)$;

(iii) real, if $\mathscr{A}=\overline{\mathbb{R}_{+}}=[0,+\infty]$ with the natural ordering, and $\beta(\mathscr{D})<+\infty$ for every bounded set $\mathscr{D} \in$ $P(E)$.

Among the known examples of MNC satisfying all the above properties we can consider the Hausdorff $M N C$

$$
\chi(\mathscr{D})=\inf \{\varepsilon>0: \mathscr{D} \text { has a finite } \varepsilon \text {-net }\} .
$$

and the Kuratowski MNC

$$
\alpha(\mathscr{D})=\inf \{\delta>0: \mathscr{D} \text { has a finite partition with sets of diameter less than } \delta\} .
$$


We can consider also an example of an MNC defined on bounded subsets of the space $C([a, b] ; \mathscr{E})$, where $\mathscr{E}$ is a Banach space. For a bounded $\mathscr{D} \subset \mathscr{E}$, set

$$
v(\mathscr{D})=\max _{\mathscr{D}^{\prime} \in \Delta(\mathscr{D})}\left(\sup _{t \in[a, b]} \chi\left(\mathscr{D}^{\prime}(t)\right), \bmod _{C}\left(\mathscr{D}^{\prime}\right)\right),
$$

where $\Delta(\mathscr{D})$ is the collection of all denumerable subsets of $\mathscr{D}, \chi$ is the Hausdorff MNC in $\mathscr{E}, \mathscr{D}^{\prime}(t)=$ $\left\{d(t): d \in \mathscr{D}^{\prime}\right\}$, and

$$
\bmod _{C}\left(\mathscr{D}^{\prime}\right)=\lim _{\delta \rightarrow 0} \sup _{d \in \mathscr{D}^{\prime}} \max _{\left|t_{1}-t_{2}\right| \leq \delta}\left\|d\left(t_{1}\right)-d\left(t_{2}\right)\right\|
$$

is the modulus of equicontinuity of the set $\mathscr{D}^{\prime}$ (see [11]). The range for the MNC $v$ is the cone $\mathbb{R}_{+}^{2}$ and max is taken in the sense of the ordering induced by this cone.

Let now $U$ be an open subset of $E, \beta$ a MNC in $E$.

Definition 2.12. A u.s.c. multimap $\mathscr{F}: \bar{U} \rightarrow K(E)$ or a u.s.c. family of multimaps $\mathscr{H}: \bar{U} \times[0,1] \rightarrow$ $K(E)$ is called $\beta$-condensing provided for every $\mathscr{D} \subseteq \bar{U}$ that is not relatively compact we have, respectively,

$$
\beta(\mathscr{F}(\mathscr{D})) \nsupseteq \beta(\mathscr{D})
$$

or, respectively,

$$
\beta(\mathscr{H}(\mathscr{D} \times[0,1])) \nsupseteq \beta(\mathscr{D}) .
$$

Definition 2.13. A closed convex subset $T \subset E$ is called fundamental for a multimap $\mathscr{F}: \bar{U} \rightarrow K(E)$ if

(1) $\mathscr{F}(\bar{U} \cap T) \subseteq T$;

(2) $x \in \overline{c o}(\mathscr{F}(x) \cup T)$ implies $x \in T$.

Let us mention the following properties of fundamental sets (see [11]).

Proposition 2.6. $\quad(i)$ The fixed point set Fix $\mathscr{F}$ is contained in each fundamental set of $\mathscr{F}$.

(ii) If $\left\{T_{\alpha}\right\}$ is a system of fundamental sets of a multimap $\mathscr{F}$ then the set $\cap_{\alpha} T_{\alpha}$ is also fundamental.

Proposition 2.7. Each $\beta$-condensing multimap $\mathscr{F}: \bar{U} \rightarrow K(E)$, where $\beta$ is a monotone, nonsingular $M N C$, admits a nonempty compact fundamental set.

\section{A RANDOM FIXED POINT INDEX FOR CONDENSING MULTIMAPS}

Let $E$ be a Banach space and let $U$ be an open subset of $E$. Let $\beta$ be a monotone nonsingulat MNC in $E$, and let $\Omega$ be a complete measurable space. Our purpose is to define the fixed point index for a multimap $\mathscr{F}: \Omega \times \bar{U} \rightarrow K(E)$ which satisfies the following conditions:

$(\mathscr{F} 1) \mathscr{F}$ is a random $u$-multimap;

$(\mathscr{F} 2)$ for every $\omega \in \Omega, \mathscr{F}(\omega, \cdot): \bar{U} \rightarrow K(E)$ is a $\beta$-condending $J^{c}$-multimap;

$(\mathscr{F} 3) x \notin \mathscr{F}(\omega, x)$ for all $(\omega, x) \in \Omega \times \partial U$.

In the sequel, the class of multimaps $\mathscr{F}$ satisfying conditions $(\mathscr{F} 1)-(\mathscr{F} 3)$ will be denoted $J_{\beta}^{c}(\Omega, \bar{U} ; E)$. Respectively, we will denote by $\widetilde{J}_{\beta}^{c}(\Omega, \bar{U} ; E)$ the collection of multimaps obeying $(\mathscr{F} 1)-(\mathscr{F} 2)$. 
Let $\mathscr{F} \in J_{\beta}^{c}(\Omega, \bar{U} ; E)$. For a fixed $\omega \in \Omega$, let $T^{\omega}$ be a nonempty compact fundamental set of the multimap $\mathscr{F}(\omega, \cdot)$ with the decomposition $D_{\mathscr{F}(\omega, \cdot)}=\left(\mathscr{F}_{n} \circ \ldots \circ \mathscr{F}_{1}\right)$. Take any retraction $\rho: E \rightarrow T^{\omega}$ and consider the multimap

$$
\rho \circ \mathscr{F}(\omega, \cdot): \bar{U} \rightarrow K(E) .
$$

It is clear that $\rho \circ \mathscr{F}(\omega, \cdot)$ is a compact $J^{c}$-multimap with the decomposition

$$
D_{\rho \circ \mathscr{F}(\omega, \cdot)}=\left(\rho \circ \mathscr{F}_{n} \circ \ldots \circ \mathscr{F}_{1}\right) .
$$

Moreover, it follows from Proposition 2.6 (i) that

$$
\operatorname{Fix}(\rho \circ \mathscr{F}(\omega, \cdot))=\operatorname{Fix} \mathscr{F}(\omega, \cdot) .
$$

Hence the fixed point index $\operatorname{ind}\left(D_{\rho \circ \mathscr{F}(\omega, \cdot)}, \bar{U}\right)$ is well defined.

Lemma 3.1. The fixed point index ind $\left(D_{\rho \circ \mathscr{F}(\omega, \cdot)}, \bar{U}\right)$ depends neither on the choice of a fundamental set $T^{\omega}$ nor on the choice of a retraction $\rho$.

Proof. Consider two nonempty compact fundamental sets $T_{0}^{\omega}$ and $T_{1}^{\omega}$ for $\mathscr{F}(\omega, \cdot)$ with retractions $\rho_{0}: E \rightarrow T_{0}^{\omega}$ and $\rho_{1}: E \rightarrow T_{1}^{\omega}$ respectively.

If $T_{0}^{\omega} \cap T_{1}^{\omega}=\emptyset$, then

$$
\operatorname{Fix}\left(\rho_{0} \circ \mathscr{F}(\omega, \cdot)\right)=\operatorname{Fix}\left(\rho_{1} \circ \mathscr{F}(\omega, \cdot)\right)=\operatorname{Fix} \mathscr{F}(\omega, \cdot)=\emptyset .
$$

Using Proposition 2.3, we have

$$
\operatorname{ind}\left(D_{\rho_{0} \circ \mathscr{F}(\omega, \cdot)}, \bar{U}\right)=\operatorname{ind}\left(D_{\rho_{1} \circ \mathscr{F}(\omega, \cdot)}, \bar{U}\right)=0 .
$$

Let $T_{0}^{\omega} \cap T_{1}^{\omega} \neq \emptyset$. From Proposition 2.6 (ii), we may assume without loss of generality that $T_{0}^{\omega} \subseteq T_{1}^{\omega}$.

Define the map $h: E \times[0,1] \rightarrow E$

$$
h(y, \lambda)=\rho_{1}\left(\lambda y+(1-\lambda) \rho_{0} y\right)
$$

and consider the multimap $\mathscr{H}: \bar{U} \times[0,1] \rightarrow K(E)$ given as

$$
\mathscr{H}(x, \lambda)=h(\mathscr{F}(\omega, x), \lambda) .
$$

It is easy to see that the compact multimap $\mathscr{H}$ belongs to the class $J^{c}(\bar{U} \times[0,1], E)$. Moreover, it realizes the homotopy of decompositions $D_{\rho_{0} \circ \mathscr{F}(\omega, \cdot)}$ and $D_{\rho_{1} \circ \mathscr{F}(\omega, \cdot)}$. In fact, it is necessary to verify only that

$$
x \notin \mathscr{H}(x, \lambda), \quad \forall(x, \lambda) \in \partial U \times[0,1] .
$$

To the contrary, suppose that there exists $(x, \lambda) \in \partial U \times[0,1]$ such that

$$
x=\rho_{1}\left(\lambda y+(1-\lambda) \rho_{0} y\right)
$$

for some $y \in \mathscr{F}(\omega, x)$. Then $x \in T_{1}^{\omega}$ and hence $y \in T_{1}^{\omega}$. Since $\rho_{0} y \in T_{1}^{\omega}$, we get

$$
x=\lambda y+(1-\lambda) \rho_{0} y,
$$

which implies $x \in \overline{c o}\left(\mathscr{F}(\omega, x) \cup T_{0}^{\omega}\right)$. This yields $x \in T_{0}^{\omega}, y \in T_{0}^{\omega}$ and $x=y$. This contradicts condition ( $\mathscr{F} 3)$.

Now the assertion follows from Proposition 2.4. This completes the proof.

The above assertion makes consistent the following notions. 
Definition 3.1. For a given $\omega \in \Omega$, the fixed point index of the decomposition of $\mathscr{F}(\omega, \cdot)$ is defined in the following way:

$$
\operatorname{ind}\left(D_{\mathscr{F}(\omega, \cdot)}, \bar{U}\right):=\operatorname{ind}\left(D_{\rho \circ \mathscr{F}(\omega, \cdot)}, \bar{U}\right),
$$

where $\rho$ is a retraction on an arbitrary nonempty compact fundamental set of $\mathscr{F}(\omega, \cdot)$.

Definition 3.2. For a given $\mathscr{F} \in J_{\beta}^{c}(\Omega, \bar{U} ; E)$ the fixed point index is defined as the following collection of numbers:

$$
\operatorname{Ind}(\mathscr{F}, \bar{U})=\left\{\operatorname{ind}\left(D_{\mathscr{F}(\omega, \cdot)}, \bar{U}\right): \omega \in \Omega\right\} .
$$

By definition, we set $\operatorname{Ind}(\mathscr{F}, \bar{U}) \neq 0$ provided $\operatorname{ind}\left(D_{\mathscr{F}(\omega, \cdot)}, \bar{U}\right) \neq 0$ for all $\omega \in \Omega$.

We now describe the main properties of the defined characteristic. Propositions 2.1 and 2.3 yield the following assertion.

Proposition 3.1. (Existence) If Ind $(\mathscr{F}, \bar{U}) \neq 0$ then $\mathscr{F}$ has a random fixed point.

Definition 3.3. Two multimaps $\mathscr{F}, \mathscr{G} \in J_{\beta}^{c}(\Omega, \bar{U} ; E)$ are said to be homotopic,

$$
\mathscr{F} \sim \mathscr{G}
$$

provided that there exists a family $\mathscr{H}: \Omega \times \bar{U} \times[0,1] \rightarrow K(E)$ such that:

$(\mathscr{H} 1)$ for each $\lambda \in[0,1], \mathscr{H}(\cdot, \cdot, \lambda): \Omega \times \bar{U} \rightarrow K(E)$ is a random $u$-multimap;

$(\mathscr{H} 2)$ for each $\omega \in \Omega$, the family $\mathscr{H}(\omega, \cdot, \cdot): \bar{U} \times[0,1] \rightarrow K(E)$ is $\beta$-condensing;

$(\mathscr{H} 3)$ for each $\omega \in \Omega$, the decompositions $D_{\mathscr{H}(\omega,, 0)}=D_{\mathscr{F}(\omega, \cdot)}$ and $D_{\mathscr{H}(\omega, \cdot, 1)}=D_{\mathscr{G}(\omega, \cdot)}$ are homotopic in the sense of Definition 2.10.

Proposition 3.2. (Homotopy invariance) If $\mathscr{F} \sim \mathscr{G}$, then

$$
\operatorname{Ind}(\mathscr{F}, \bar{U})=\operatorname{Ind}(\mathscr{G}, \bar{U}) .
$$

Proof. For a given $\omega \in \Omega$, there exists a nonempty compact convex set $T \subset E$ which is fundamental for each multimap $\mathscr{H}(\omega, \cdot, \lambda), \lambda \in[0,1]$ (Theorem 2.2.1 of [11]). Let $\rho: E \rightarrow T$ be any retraction. Then it is easy to verify that compact decompositions

$$
D_{\rho \circ \mathscr{H}(\omega, \cdot, 0)}=D_{\rho \circ \mathscr{F}(\omega, \cdot)}
$$

and

$$
D_{\rho \circ \mathscr{H}(\omega, \cdot, 1)}=D_{\rho \circ \mathscr{G}(\omega, \cdot)}
$$

are homotopic. Using Proposition 2.4, we obtain that

$$
\operatorname{ind}\left(D_{\mathscr{F}(\omega, \cdot)}, \bar{U}\right)=\operatorname{ind}\left(D_{\mathscr{G}(\omega, \cdot)}, \bar{U}\right),
$$

which yields the desired conclusion. This completes the proof.

The next property follows directly from Proposition 2.5.

Proposition 3.3. (Additivity) For a given $\mathscr{F} \in J_{\beta}^{c}(\Omega, \bar{U} ; E)$, let, for every $\omega \in \Omega$,

$$
\operatorname{Fix} \mathscr{F}(\omega, \cdot) \cap U \subset \bigcup_{j=1}^{k} U_{j},
$$


where $U_{j}, j=1, \ldots, k$ are open disjoint in $U$. Then

$$
\operatorname{Ind}(\mathscr{F}, \bar{U})=\left\{\sum_{j=1}^{k} \operatorname{ind}\left(D_{\mathscr{F}(\omega, \cdot)}, \bar{U}_{j}\right): \omega \in \Omega\right\} .
$$

As an application of the existence principle, we consider the following fixed point result.

Theorem 3.1. Let $\mathscr{F} \in J_{\beta}^{c}(\Omega, \bar{U} ; E)$, where $U$ is convex neighborhood of the origin and

$$
x \notin \lambda F(\omega, x), \quad \forall \omega \in \Omega, x \in \partial U, 0 \leq \lambda<1 .
$$

Then $\mathscr{F}$ has a random fixed point.

Proof. Define the family $\mathscr{H}: \Omega \times \bar{U} \times[0,1] \rightarrow K(E)$ in the following way. For a given $\omega \in \Omega$, let

$$
D_{\mathscr{F}(\omega, \cdot)}=\left(\mathscr{F}_{n} \circ \ldots \circ \mathscr{F}_{1}\right)
$$

be a decomposition of $\mathscr{F}(\omega, \cdot)$. Define

$$
\mathscr{H}(\omega, x, \lambda)=\left(\psi \circ \mathscr{F}_{n}^{\star} \circ \ldots \circ \mathscr{F}_{1}^{\star}\right)
$$

where, for $1 \leq i \leq n$,

$$
\mathscr{F}_{i}^{\star}(\cdot, \lambda)=\mathscr{F}_{i} \times\{\lambda\}, \quad \lambda \in[0,1]
$$

and

$$
\psi(z, \lambda)=\lambda x, \quad \lambda \in[0,1] .
$$

It is clear that the family $\mathscr{H}$ satisfies conditions $(\mathscr{H} 1)$ and $(\mathscr{H} 3)$ of Definition 3.2.

To verify condition $(\mathscr{H} 2)$, we denote, for $\omega \in \Omega, \mathscr{H}_{\omega}=\mathscr{H}(\omega, \cdot, \cdot)$ and suppose that

$$
\left.\beta\left(\mathscr{H}_{\omega}(\mathscr{D} \times[0,1])\right)\right) \geq \beta(\mathscr{D})
$$

for some $\mathscr{D} \subset \bar{U}$.

Since

$$
\left.\mathscr{H}_{\omega}(\mathscr{D} \times[0,1])\right)=\overline{c o}(\mathscr{F}(\omega, \mathscr{D}) \cup\{0\})
$$

we have

$$
\beta(\mathscr{F}(\omega, \mathscr{D}) \geq \beta(\mathscr{D}),
$$

which implies that $\mathscr{D}$ is relatively compact.

So, the family $\mathscr{H}$ realizes the homotopy connecting the initial multimap $\mathscr{F}$ and the zero map. This means, by the normalization property of the topological degree, that $\operatorname{ind}\left(D_{\mathscr{F}(\omega, \cdot)}, \bar{U}\right)=1$ for all $\omega \in \Omega$. Hence

$$
\operatorname{Ind}(\mathscr{F}, \bar{U}) \neq 0
$$

and we apply Proposition 3.2. This completes the proof.

By using similar methods, we can prove the following assertion.

Theorem 3.2. Let $\mathscr{F} \in \widetilde{J}_{\beta}^{c}(\Omega, \bar{U} ; E)$, where open set $U$ is convex and

$$
\mathscr{F}(\Omega \times \partial U) \subset \bar{U} .
$$

Then $\mathscr{F}$ has a random fixed point. 


\section{Acknowledgments}

The authors are grateful to the reviewers for useful suggestions which improved the contents of this paper. The second author was supported by the Ministry of Education and Science of the Russian Federation in the frameworks of the project part of the state work quota (Project No. 1.3464.2017/4.6) and by the RFBR-MOST grant No. 17-51-52022.

\section{REFERENCES}

[1] J. Andres, L. Górniewicz, Random topological degree and random differential inclusions, Topol. Methods Nonlinear Anal. 40 (2012), 337-358

[2] R. Bader, W. Kryszewski, Fixed-point index for compositions of set-valued maps with proximally $\infty$-connected values on arbitrary ANR's, Set-Valued Anal. 2 (1994), 459-480.

[3] I. Beg, N. Shahzad, Random fixed points of random multivalued operators on Polish spaces, Nonlinear Anal. 20 (1993), 835-847.

[4] Y.G. Borisovich, B.D. Gelman, A.D. Myshkis, V.V. Obukhovskii, Topological methods in the theory of fixed points of multivalued mappings, (Russian) Uspekhi Mat. Nauk 35 (1980), 59-126. (English translation: Russian Math. Surveys 35 (1980), 65-143.)

[5] K. Borsuk, Theory of Retracts, Monografie Mat. 44, WN, Warszawa, 1967.

[6] H. Engl, Random fixed point theorems for multivalued mappings, Pacific J. Math. 76 (1978), 351-360.

[7] L. Górniewicz, Topological Fixed Point Theory of Multivalued Mappings, 2nd ed., Springer, Dordrecht, 2006.

[8] S. Hu, N. Papageorgiou, Handbook of Multivalued Analysis, Vol. I, Theory, Kluwer, Dordrecht, 1997.

[9] D.M. Hyman, On decreasing sequences of compact absolute retracts, Fund Math. 64 (1969), 91-97.

[10] S. Itoh, Random fixed point theorems with an application to random differential equations in Banach spaces, J. Math. Anal. Appl. 67 (1979), 261-273.

[11] M. Kamenskii, V. Obukhovskii, P. Zecca, Condensing Multivalued Maps and Semilinear Differential Inclusions, Walter de Gruyter, Berlin - New York, 2001.

[12] A.D. Myshkis, Generalizations of the theorem on a stationary point of a dynamical system inside a closed trajectory. Math. Sb. 34 (1954), 525-540. (in Russian)

[13] A. Nowak, Random fixed points of multifunctions, Prace Mat. Uniw. Ślaskiego 420 (1981), 36-41.

[14] L.E. Rybiński, Random fixed points and viable random solutions of functional-differential inclusions, J. Math. Anal. Appl. 142 (1989), 53-61.

[15] E. Tarafdar, P. Watson, X.-Z. Yuan, Random coincidence degree theory with applications to random differential inclusions, Comment. Math. Univ. Carolinae 37 (1996), 725-748.

[16] H.K. Xu, Some random fixed point theorems for condensing and nonexpansive operators, Proc. Amer. Math. Soc. 110 (1990), 395-400. 